\title{
Efficacy of heel lifts versus calf muscle eccentric exercise for mid-portion Achilles tendinopathy (HEALTHY): a randomised trial
}

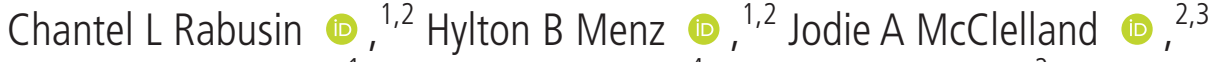

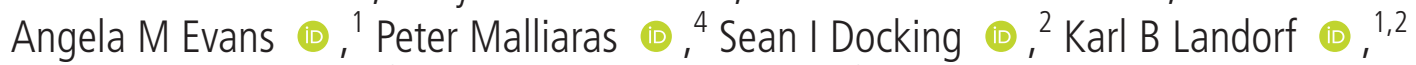 \\ James M Gerrard (1) ${ }^{1,2}$ Shannon E Munteanu (i) 1,2
}

${ }^{1}$ Discipline of Podiatry, School of Allied Health, Human Services and Sport, La Trobe University, Melbourne, Victoria, Australia 'La Trobe Sport and Exercise Medicine Research Centre, School of Allied Health, Human Services and Sport, La Trobe University, Melbourne, Victoria, Australia

${ }^{3}$ Discipline of Physiotherapy, School of Allied Health, Human Services and Sport, La Trobe University, Melbourne, Victoria, Australia

${ }^{4}$ Department of Physiotherapy, Monash University Faculty of Medicine Nursing and Health Sciences, Frankston, Victoria, Australia

Correspondence to Chantel L Rabusin, Discipline of Podiatry, School of Allied Health, Human Services and Sport, La Trobe University, Melbourne, VIC 3086, Australia;

c.rabusin@latrobe.edu.au

Accepted 17 August 2020 Published Online First 28 September 2020

\section{ABSTRACT}

Objective(s) To compare the efficacy of in-shoe heel lifts to calf muscle eccentric exercise in reducing pain and improving function in mid-portion Achilles tendinopathy. Methods This was a parallel-group randomised superiority trial at a single centre (La Trobe University Health Sciences Clinic, Discipline of Podiatry, Melbourne, Victoria, Australia). One hundred participants (52 women and 48 men, mean age 45.9, SD 9.4 years) with clinically diagnosed and ultrasonographically confirmed midportion Achilles tendinopathy were randomly allocated to either a (1) heel lifts $(n=50)$ or (2) eccentric exercise $(n=50)$ group. The primary outcome measure was the Victorian Institute of Sport Assessment-Achilles (VISA-A) questionnaire at 12 weeks. Differences between groups were analysed using intention to treat with analysis of covariance.

Results There was $80 \%$ follow-up of participants ( $n=40$ per group) at 12 weeks. The mean VISA-A score improved by 26.0 points $(95 \% \mathrm{Cl} 19.6$ to 32.4$)$ in the heel lifts group and by 17.4 points $(95 \% \mathrm{Cl} 9.5$ to 25.3$)$ in the eccentric exercise group. On average, there was a between-group difference in favour of the heel lifts for the VISA-A (adjusted mean difference $9.6,95 \% \mathrm{Cl} 1.8$ to $17.4, p=0.016)$, which approximated, but did not meet our predetermined minimum important difference of 10 points.

Conclusion In adults with mid-portion Achilles tendinopathy, heel lifts were more effective than calf muscle eccentric exercise in reducing pain and improving function at 12 weeks. However, there is uncertainty in the estimate of effect for this outcome and patients may not experience a clinically worthwhile difference between interventions.

Trial registration number ACTRN12617001225303.

\section{BACKGROUND}

Mid-portion Achilles tendinopathy is a common musculoskeletal condition, presenting as pain and stiffness between 2 and $6 \mathrm{~cm}$ above the posterior calcaneus. ${ }^{1-3}$ The pathological tendon shows abnormal changes on ultrasound imaging, such as altered tendon structure, disorganisation of fibres and thickening. ${ }^{4}$ Individuals with Achilles tendinopathy often report reduced participation in physical activity, ${ }^{6}$ impaired performance, ${ }^{7}$ and deficits in ankle joint plantarflexion strength and endurance. ${ }^{8}$ Achilles tendinopathy can also impact health-related quality of life. ${ }^{9}$ Accordingly, effective interventions are essential for this common and disabling condition.

Calf muscle eccentric exercise has been extensively researched, frequently administered, ${ }^{10-13}$ and is the recommended intervention for the management of mid-portion Achilles tendinopathy. ${ }^{10}$ Numerous eccentric exercise protocols exist, varying in load, frequency and speed. Nevertheless, similar treatment effectiveness has been reported, suggesting there is currently no optimum protocol. ${ }^{14}$ Although eccentric exercise continues to be the 'gold standard' for mid-portion Achilles tendinopathy, ${ }^{10}$ success rates are varied, with up to $44 \%$ of those affected reporting the exercise to be ineffective. ${ }^{15} 16$ One reason for the varied success could be heterogeneous exercise adherence. ${ }^{17}$

Heel lifts are inserts placed inside footwear, and are also commonly prescribed for mid-portion Achilles tendinopathy. ${ }^{18} 19$ Heel lifts do not require active engagement to exert their effects-adherence is usually high. Heel lifts reduce ankle joint dorsiflexion during running, and reduce gastrocnemius muscle length and Achilles tendon strain during walking. ${ }^{20}$ While these biomechanical findings suggest heel lifts may be effective for mid-portion Achilles tendinopathy, there have been no high quality randomised trials of this intervention. The aim of this study was, therefore, to compare heel lifts to calf muscle eccentric exercise for reducing pain and improving function in individuals with mid-portion Achilles tendinopathy.

\section{METHODS}

Study design

The Heel lifts vs calf muscle eccentric Exercise for AchiLles Tendinopat $\underline{\mathrm{HY}}$ study was a parallel-group randomised superiority trial. The description of the methods of this trial have been summarised from the published protocol. ${ }^{21}$ Findings are reported according to the Consolidated Standards of Reporting Trials 2010 Statement $^{22} 23$ and the TIDieR guide. ${ }^{24} 25$

\section{Protocol deviations}

In the trial registration and study protocol, ${ }^{21}$ we specified that we would randomise 92 participants. However, we had more eligible volunteers for study participation and therefore enrolled 100 participants into the study. 


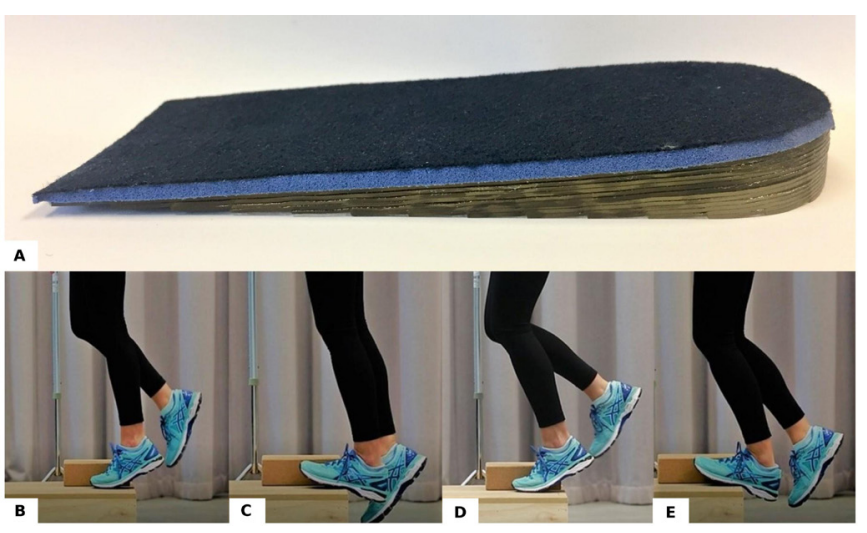

Figure 1 (A) Clearly adjustable heel lifts. (B-E) Calf muscle eccentric exercise.

\section{Study population and setting}

Between September 2017 and December 2017, participants were recruited from advertisements placed in local newspapers and on social media platforms. Full inclusion and exclusion criteria have been detailed previously; ${ }^{21}$ a summary of the eligibility criteria is below. Participants aged at least 18 years of age, with clinically diagnosed and ultrasonographically confirmed mid-portion Achilles tendinopathy, with pain present for at least 2 months and reporting a minimum level of pain of at least a 3 out of 10 on a Numerical Rating Scale-11 in the last week were included. Participants were excluded if they had previous Achilles tendon rupture or surgery in the most symptomatic limb(s), chronic ankle instability, treatment with in-shoe heel lifts or a calf muscle eccentric exercise programme in the previous 3 months, or a medical condition (eg, inflammatory arthritis) that deemed the participant unsuitable for participation. All assessments were conducted at a single centre (La Trobe University Health Sciences Clinic, Discipline of Podiatry, Melbourne, Victoria, Australia).

\section{Study interventions}

Participants allocated to the heel lifts group received three pairs for bilateral use (ie, irrespective of unilateral/bilateral midportion AT) of Clearly Adjustable $12 \mathrm{~mm}$ heel lifts (A. Algeo, Australia) made from firm (Shore A 90) multilayered clear vinyl. Heel lifts were placed in up to three pairs of each participant's most frequently worn footwear. According to each participant's shoe size, small, medium or large heel lifts were dispensed. A $3.2 \mathrm{~mm}$ PPT Ultralux top cover was adhered to the top surface to maximise comfort (figure 1A). Existing insoles were removed from participant's footwear to minimise heel slippage.

The participants allocated to the eccentric exercise group received a 12-week calf muscle eccentric exercise programme, based on the Alfredson method. ${ }^{11}$ The technique for performing this exercise programme has been detailed previously. ${ }^{21}$ The program involved performing the exercises twice daily, 7 days per week for 12 weeks. Participants with bilateral symptoms performed the programme on both sides. The programme involved two exercises: the first with the knee extended, to activate gastrocnemius (figure $1 \mathrm{~B}, \mathrm{C}$ ), and the second, with the knee flexed to increase activation of soleus (figure 1D,E). Both exercises involved three sets of 15 repetitions, performed twice daily on the symptomatic limb(s). Initially, resistance consisted of bodyweight, and the participants stood with all their bodyweight on the injured leg. Participants were advised to continue the exercise even if they experienced pain. However, they were advised to stop and resume the following day if the pain became disabling. Participants were advised to increase the resistance component of the programme once they could perform both exercises with no pain or discomfort on the injured side. Participants were told to use a weighted backpack to increase resistance, beginning with the addition of $5 \mathrm{~kg}$ of mass to the backpack, and to increase the load by $5 \mathrm{~kg}$ increments.

Both groups received a handout explaining their allocated intervention and a modified physical activity programme based on the pain-monitoring model, ${ }^{13}$ as detailed previously. ${ }^{21}$

\section{Treatment preference, credibility and expectancy}

Participants were asked prior to randomisation to indicate their preferred treatment. ${ }^{26}$ Given that participants were not blinded to their allocated intervention, their beliefs about treatment were considered important, so participants completed the Credibility/Expectancy Questionnaire (CEQ) ${ }^{27}$ after being allocated to an intervention group.

\section{Outcome measures}

Outcome measures were collected at 2, 6 and 12 weeks unless stated otherwise. The primary end-point was at 12 weeks. To minimise participant burden, a postal questionnaire was used for the assessment at 6 weeks.

\section{Primary outcome measure}

The primary outcome was the Victorian Institute of Sport Assessment-Achilles (VISA-A) questionnaire. The VISA-A is a reliable self-administered patient-reported outcome measure containing eight questions evaluating pain, function and activity. ${ }^{28}$ The maximum obtainable score is 100 , with a lower score indicating greater clinical severity. Participants with bilateral symptoms completed the questionnaire on the most painful side (or right side, if Achilles tendons were equally painful).

\section{Secondary outcome measures}

Secondary outcome measures included the following.

1. Achilles tendon structure using ultrasound tissue characterisation (UTC) and anterior-posterior thickness at baseline and 12 weeks, as described previously. ${ }^{21}$ Tendon structure was quantified by the percentage of echo-types: aligned fibrillar structure (AFS; echo types I and II) and disorganised structure (DIS; echo types III and IV). ${ }^{29} 30$

2. Severity of pain (at worst in the previous week) measured on a $100 \mathrm{~mm}$ Visual Analogue Scale (VAS). ${ }^{31}$

3. Health status (using the EuroQol 5 Dimension 5 Level (EQ5D-5L) questionnaire) was expressed for both of its components, the index value (scores range from $<0$ (worse than dead) to 0 (dead) to 1 (full health)) and the VAS value (scores range from 0 (worst imaginable health state) to 100 (best imaginable health state)). ${ }^{32}$

4. Magnitude of physical activity in the previous week (using the 7-day Recall Physical Activity Questionnaire). ${ }^{33}$

5. Calf muscle function at baseline and 12 weeks (using the standing heel rise test, assessed on flat ground). ${ }^{34}$ This test recorded the maximum number of repetitive unilateral weightbearing raises (concentric-eccentric muscle action) that could be performed until fatigue, and was conducted to the sound of a metronome (one heel rise every $2 \mathrm{~s}$ with a metronome set at 60 beats per minute).

6. Self-reported treatment effectiveness for pain and function (using the Patient Impression of Change (PIC) questionnaire). ${ }^{31}$ This variable was then dichotomised into the categories of 'effective' ('much improved' or 'very much im- 
proved') and 'ineffective' ('minimally improved' to 'very much worse'). ${ }^{35}$

7. Use of paracetamol rescue medication and cointerventions to relieve Achilles pain.

\section{Intervention adherence}

Adherence to the interventions was measured at 2, 6 and 12 weeks via questionnaires. Participants in the heel lifts group were asked to report the number of days and hours per day they had worn the heel lifts in the preceding period. Similarly, participants in the eccentric exercise group were asked to report the number of days per week they had performed the exercise programme in each week of the preceding period.

\section{Adverse events}

Adverse events were measured at 2, 6 and 12 weeks via questionnaires. Participants were asked to document the type of adverse event, the body location, the frequency and/or severity of the effect. $^{36}$

\section{Randomisation and blinding}

To ensure allocation concealment, randomisation was provided by an independent computer (online) generated service (Griffith University, Queensland, Australia). The allocation for each participant was provided once eligibility was confirmed and baseline measures were completed. To ensure balance of participants in both groups, the random allocation sequence was generated with a 1:1 allocation using permuted blocks of random sizes $(2,4,6)$. Due to the nature of the interventions, neither the participants, nor research staff administering the interventions (CLR and JMG), assessing outcomes (CLR), or entering data (CLR) were blinded to group allocation. However, data entry was independently validated, and research staff analysing data (CLR, HBM and JMG) were blinded to group allocation (masking used).

\section{Sample size}

An a priori sample size calculation estimated that a total of 92 participants (46 per group) would be needed to detect a difference between two groups, based on a power of $80 \%$, minimal important difference (MID) of 10 points in the VISA-A, ${ }^{16} \mathrm{SD}$ of $16.9,{ }^{37}$ standardised effect size of 0.59 and an alpha of 0.05 . We did not allow for participant loss to follow-up in our calculation as we used multiple imputation for missing data. ${ }^{38}$

\section{Data analysis}

SPSS V.26 (IBM) was used for statistical analysis using the intention-to-treat principle. All data were explored for normality, and non-normally distributed data were transformed where possible. Data unable to be transformed were analysed using non-parametric tests. To avoid overtesting and reduce the risk of type I-associated errors, ${ }^{39} 40$ the end-point was the completion of the study (12 weeks). Multiple imputation was used to replace missing data using five iterations, with sex, age, baseline scores and group allocation used as predictors, ${ }^{38}$ for all primary and secondary outcome measures. The exception was for the variables; self-reported treatment effectiveness, use of cointerventions, adherence and adverse events, where data substitution was not applied. Continuously scaled measures were analysed using analysis of covariance (ANCOVA) with baseline scores and the intervention group as the independent variable. ${ }^{41}$ For dichotomous-scaled outcome measures, relative risk, risk difference and number needed to treat (NNT) were used for analysis.
Where appropriate, 95\% CIs and p values were calculated to complement point estimates. The participants' expectations (how much they expected they would benefit from the treatment) and credibility (beliefs about the logic of an intervention), measured using the CEQ at baseline, were compared between groups using the Mann-Whitney U (for non-normally distributed data) and independent t-tests (for normally distributed data).

\section{RESULTS}

\section{Participants}

Between September 2017 and December 2017, 368 participants contacted the researcher (CLR) for information regarding the trial. One hundred participants were enrolled into the study. Fifty participants were allocated to the heel lifts group and 50 participants were allocated to the eccentric exercise group. There were no clinically relevant differences in baseline characteristics between groups (table 1). Overall, 83, 73 and 80 participants completed the 2, 6 and 12 weeks follow-up assessments, respectively (figure 2).

\section{Primary outcome measure}

There was $80 \%$ follow-up of participants ( $n=40$ per group) at 12 weeks. The mean VISA-A score improved by 26.0 points (95\% CI 19.6 to 32.4 ) in the heel lifts group and by 17.4 points (95\% CI 9.5 to 25.3 ) in the eccentric exercise group. On average, there was a between-group difference in favour of the heel lifts for the VISA-A (adjusted mean difference 9.6, 95\% CI 1.8 to 17.4, $\mathrm{p}=0.016)$.

\section{Secondary outcome measures}

At 12 weeks, there were no statistically significant betweengroup differences in Achilles anterior-posterior diameter or Achilles tendon integrity (AFS and DIS) assessed using UTC (table 2). However, there was a statistically significant betweengroup difference for severity of pain (VAS) (adjusted mean difference $-19.2,95 \% \mathrm{CI}-31.0$ to $-7.4, \mathrm{p}=0.002$ ) in favour of the heel lifts. There was also a statistically significant betweengroup difference for the EQ-5D-5L index score (adjusted mean difference $0.1,95 \%$ CI 0.0 to $0.2, p=0.044$ ) in favour of the heel lifts. There were no statistically significant between-group differences for EQ-5D-5L VAS, level of physical activity or calf muscle function. Participants in the heel lifts group were more likely to report an improvement in pain (RR 1.4 , 95\% CI 1.0 to 2.0, NNT 4) and function (RR 1.6, 95\% CI 1.0 to 2.3, NNT 4), as measured using the PIC (online supplemental file 1).

\section{Treatment preference, credibility and expectancy}

Fifty-two per cent of participants in the heel lifts group and 64\% of participants in the eccentric exercise group had a preference for the eccentric exercise programme (table 1). Participants in both groups considered their interventions to be credible and they expected to benefit from them (online supplemental file 2). There were no statistically significant between-group differences regarding the credibility and expectancy of treatment.

\section{Intervention adherence}

Table 3 shows the adherence at each time point for both treatment groups. Adherence was greater in the heel lifts group at all time periods.

\section{Use of rescue medication and cointerventions}

Overall, seven participants reported using cointerventions or rescue medication (paracetamol) during the study (online 


\begin{tabular}{|c|c|c|}
\hline & $\begin{array}{l}\text { Heel lifts group } \\
(\mathrm{n}=50)\end{array}$ & $\begin{array}{l}\text { Eccentric exercise } \\
\text { group }(n=50)\end{array}$ \\
\hline \multicolumn{3}{|l|}{ Demographics and anthropometrics } \\
\hline Age, years & $46.1(9.0)$ & $45.6(9.8)$ \\
\hline Female* & $26(52)$ & $26(52)$ \\
\hline Height, $\mathrm{cm}$ & $171.9(9.1)$ & $172.6(10.7)$ \\
\hline Weight, kg & $85.6(17.7)$ & $89.9(22.0)$ \\
\hline Body mass index, $\mathrm{kg} / \mathrm{m}^{2} \dagger$ & $28.0(24.3$ to 31.8$)$ & $28.8(26.3$ to 31.1$)$ \\
\hline Waist to hip ratio & $0.9(0.1)$ & $0.9(0.1)$ \\
\hline FPI scoret & $3(2$ to 4$)$ & $3(0$ to 4$)$ \\
\hline Supinated (FPI -12 to 0$)^{*}$ & $10(20)$ & $15(30)$ \\
\hline Normal (FPI 1 to 7)* & $40(80)$ & $34(68)$ \\
\hline Pronated (FPI 8 to 12$)^{*}$ & $0(0)$ & $1(2)$ \\
\hline $\begin{array}{l}\text { Ankle joint dorsiflexion range of motion, } \\
\text { degrees } \neq\end{array}$ & $38.5(6.5)$ & $39.1(7.8)$ \\
\hline \multicolumn{3}{|l|}{ Self-reported education* } \\
\hline Primary school completed & 1 (2) & $3(6)$ \\
\hline High school (or equivalent) completed & $14(28)$ & 7 (14) \\
\hline Vocational training & $11(22)$ & $12(24)$ \\
\hline College/university completed & $15(30)$ & $15(30)$ \\
\hline Postgraduate degree completed & $9(18)$ & $12(24)$ \\
\hline Don't know & $0(0)$ & $1(2)$ \\
\hline \multicolumn{3}{|l|}{ Self-reported medical conditions* } \\
\hline Asthma & $4(8)$ & $0(0)$ \\
\hline Hypercholesterolaemia & $0(0)$ & 2 (4) \\
\hline Hypertension & $10(20)$ & $8(16)$ \\
\hline Leg cramps & $10(20)$ & $4(8)$ \\
\hline Osteoarthritis & $4(8)$ & $6(12)$ \\
\hline Hands & 1 (2) & $1(2)$ \\
\hline Knee & $3(6)$ & $5(10)$ \\
\hline Feet & 2 (4) & $0(0)$ \\
\hline Other & $4(8)$ & $9(18)$ \\
\hline \multicolumn{3}{|l|}{ Self-reported medications* } \\
\hline Uses at least 1 medication & $20(40)$ & $28(56)$ \\
\hline \multicolumn{3}{|l|}{ Physical activity } \\
\hline Partakes in physical activity* & $43(86)$ & $41(82)$ \\
\hline $\begin{array}{l}\text { Average hours of physical activity }{ }^{\text {p per }} \\
\text { week, hours } \dagger\end{array}$ & 4.5 (1.9 to 7.7$)$ & 5.1 (0.9 to 8.2$)$ \\
\hline \multicolumn{3}{|l|}{ Footwear } \\
\hline Women's shoe size, USA† & $8.0(7.5$ to 9.0$)$ & $8.0(8.0$ to 10.0$)$ \\
\hline Men's shoe size, USAT & $10.0(9.0$ to 11.0$)$ & $10.5(10.0$ to 11.0$)$ \\
\hline Athletic shoe worn most frequently* & $20(40)$ & $21(42)$ \\
\hline Rearfoot height, $\mathrm{mm} \dagger$ & $31.6(26.0$ to 37.0$)$ & 33.1 (26.8 to 36.9$)$ \\
\hline Forefoot height, $\mathrm{mm} \dagger$ & 19.1 (13.0 to 23.4$)$ & $20.6(12.9$ to 24.1$)$ \\
\hline Pitch, mmt & $12.3(8.4$ to 15.8$)$ & $12.5(9.7$ to 16.7$)$ \\
\hline Current use of foot orthoses* & $6(12)$ & $12(24)$ \\
\hline Current use of ankle brace* & $1(2)$ & $1(2)$ \\
\hline \multicolumn{3}{|l|}{ Achilles tendinopathy characteristics } \\
\hline Left/right/bilateral* & $\begin{array}{l}11(22) / 14(28) / 25 \\
(50)\end{array}$ & $10(20) / 7(14) / 33(66)$ \\
\hline Duration of symptoms, months $\dagger$ & $18.0(6.0$ to 36.0$)$ & $22.5(8.0$ to 36.0$)$ \\
\hline \multicolumn{3}{|l|}{ Outcome measures } \\
\hline VISA-A & $57.0(15.4)$ & $53.3(17.1)$ \\
\hline Achilles tendon AP diameter, $\mathrm{mm}$ & $8.61(1.88)$ & $9.83(2.12)$ \\
\hline Achilles tendon integrity: AFS, \% & $76.3(17.6)$ & $71.7(19.6)$ \\
\hline Achilles tendon integrity: DIS, \% & $23.7(17.6)$ & $28.3(19.6)$ \\
\hline Severity of pain (VAS), mm & $56.1(24.6)$ & $60.1(21.9)$ \\
\hline EQ-5D-5L, Index & $0.76(0.11)$ & $0.73(0.11)$ \\
\hline EQ-5D-5L, VAS & $76.1(12.7)$ & $77.4(15.4)$ \\
\hline $\begin{array}{l}\text { Self-reported Physical Activity Recall } \\
\text { (PAR), kilocalories per dayt }\end{array}$ & 3493 (2916 to 4118) & 3688 (3197 to 4497) \\
\hline
\end{tabular}

Continued
Table 1 Continued

\begin{tabular}{cll}
\hline & $\begin{array}{l}\text { Heel lifts group } \\
(\mathbf{n}=50)\end{array}$ & $\begin{array}{l}\text { Eccentric exercise } \\
\text { group }(\mathbf{n}=50)\end{array}$ \\
\hline $\begin{array}{c}\text { Calf muscle function, repetitions } \\
\text { Preferred treatment* }\end{array}$ & $18.9(10.6)$ & $17.9(11.0)$ \\
\hline Preferred HL/EE/impartial & $\begin{array}{l}19(38) / 26(52) / 5 \\
(10)\end{array}$ & $12(24) / 32(64) / 6(12)$ \\
Received preferred intervention & $19(38)$ & $32(64)$ \\
\hline
\end{tabular}

EQ-5D-5L, EuroQol 5 dimension 5 level questionnaire was expressed for both of its components, the index value (scores range from $<0$ (worse than dead) to 0 (dead) to 1 (full health)) and the VAS value (scores range from 0 (worst imaginable health state) to 100 (best imaginable health state)). PAR questionnaire. Values are mean (SD) unless otherwise noted. ${ }^{*} \mathrm{n}(\%)$.

tMedian (IQR), where IQR is expressed as Q1-03 (non-normally distributed data).

¥Measured using the weightbearing lunge technique. Details of the measurements can be found in the study protocol. ${ }^{21}$

AFS, aligned fibrillar structure; $\mathrm{AP}$, anterior-posterior; DIS, disorganised structure; $\mathrm{EE}$, eccentric exercise; FPI, Foot Posture Index; HL, heel lifts; VAS, Visual Analogue Scale; VISA-A, Victorian Institute of Sports Assessment-Achilles.

supplemental file 3). There were no statistically significant between-group differences in the proportion of participants who reported using a cointervention or rescue medication. At 2 weeks, one participant in the eccentric exercise group reported using ice and one participant reported using an oral non-steroidal anti-inflammatory agent. At 6 weeks, one participant in the heel lifts group reported using rescue medication (paracetamol plus codeine) and one participant in the eccentric exercise group reported using a self-massaging machine. At 12 weeks, three participants in the eccentric exercise group reported using a cointervention: one participant reported performing

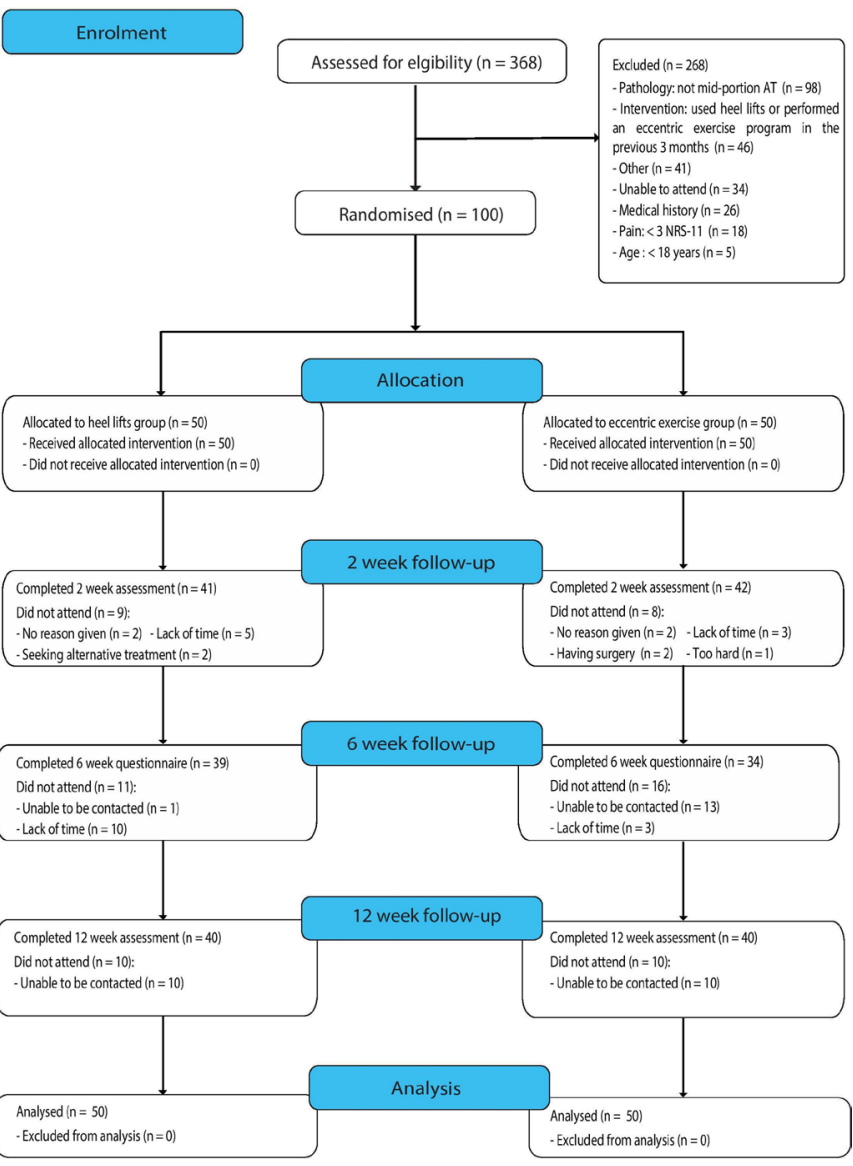

Figure 2 Flow of participants through the study. AT, Achilles tendon; NRS, Numerical Rating Scale. 
Table 2 Primary and secondary outcome measures

\begin{tabular}{|c|c|c|c|c|}
\hline \multirow[b]{2}{*}{ Outcome measure } & \multicolumn{2}{|c|}{ Group } & \multirow[b]{2}{*}{ Adjusted mean differencet* } & \multirow[b]{2}{*}{$P$ value } \\
\hline & Heel lifts* & Eccentric exercise* & & \\
\hline \multicolumn{5}{|l|}{ VISA-A } \\
\hline Baseline & $57.0(15.4)$ & $53.3(17.1)$ & - & - \\
\hline 2 weeks & $69.3(14.1)$ & $58.9(19.0)$ & - & - \\
\hline 6 weeks & $71.9(16.8)$ & $64.2(20.3)$ & - & - \\
\hline 12 weeks & $83.0(16.9)$ & $70.7(22.2)$ & 9.61 (1.83 to 17.40$)$ & 0.016 \\
\hline \multicolumn{5}{|c|}{ Achilles tendon AP diameter, mm } \\
\hline Baseline & $8.61(1.88)$ & $9.83(2.12)$ & - & - \\
\hline 12 weeks & $8.31(2.23)$ & $8.98(2.32)$ & $3.82(-1.43$ to 9.08$)$ & $0.865 \S$ \\
\hline \multicolumn{5}{|c|}{ Achilles tendon integrity: AFS, \%¥ } \\
\hline Baseline & $76.3(17.6)$ & $71.7(19.6)$ & - & - \\
\hline 12 weeks & $74.1(18.2)$ & $69.7(21.0)$ & $3.41(-6.34$ to 13.14$)$ & 0.464 \\
\hline \multicolumn{5}{|c|}{ Achilles tendon integrity: DIS, \% } \\
\hline Baseline & $23.7(17.6)$ & $28.3(19.6)$ & & \\
\hline 12 weeks & $26.9(16.6)$ & $30.0(28.5)$ & $-2.18(-14.15$ to 9.78$)$ & 0.8419 \\
\hline \multicolumn{5}{|c|}{ Severity of pain (VAS), mm } \\
\hline Baseline & $56.1(24.6)$ & $60.1(21.9)$ & - & - \\
\hline 2 weeks & $40.5(26.2)$ & $49.3(25.6)$ & - & - \\
\hline 6 weeks & $24.2(23.8)$ & $39.3(29.1)$ & - & - \\
\hline 12 weeks & $18.1(23.2)$ & $37.6(31.1)$ & $-19.18(-31.00$ to -7.36$)$ & 0.0029 \\
\hline \multicolumn{5}{|l|}{ EQ-5D-5L, Index } \\
\hline Baseline & $0.76(0.11)$ & $0.73(0.11)$ & - & - \\
\hline 2 weeks & $0.81(0.10)$ & $0.77(0.15)$ & - & - \\
\hline 6 weeks & $0.79(0.15)$ & $0.76(0.21)$ & - & - \\
\hline 12 weeks & $0.87(0.13)$ & $0.81(0.12)$ & $0.10(0.02$ to 0.18$)$ & 0.044 \\
\hline \multicolumn{5}{|l|}{ EQ-5D-5L, VAS } \\
\hline Baseline & $76.1(12.7)$ & $77.4(15.4)$ & - & - \\
\hline 2 weeks & $82.5(10.8)$ & $79.9(14.8)$ & - & - \\
\hline 6 weeks & $80.0(14.3)$ & $80.0(17.9)$ & - & - \\
\hline 12 weeks & $84.9(11.4)$ & $82.8(13.2)$ & $-0.89(-2.21$ to 0.44$)$ & $0.189^{* *}$ \\
\hline \multicolumn{5}{|c|}{$\begin{array}{l}\text { Self-reported physical activity (PAR), kilocalories } \\
\text { per dayt† }\end{array}$} \\
\hline Baseline & 3440 (2916 to 4118$)$ & 3650 (3197 to 4497$)$ & - & - \\
\hline 2 weeks & 3230 (2662.9 to 4065$)$ & 3377 (3007 to 4526.2 ) & - & - \\
\hline 6 weeks & 1846 (1669.1 to 2287$)$ & 1855 (1653.4 to 2348.1 ) & - & - \\
\hline 12 weeks & 1856.5 (1568 to 2232 ) & 1908 (1558 to 2417$)$ & - & $0.799 \ddagger \ddagger$ \\
\hline \multicolumn{5}{|c|}{ Calf muscle function, repetitions } \\
\hline Baseline & $18.9(10.6)$ & $17.9(11.2)$ & & \\
\hline 12 weeks & $20.1(8.8)$ & $21.6(10.3)$ & -1.76 (-5.65 to 2.13$)$ & 0.6249 \\
\hline \multicolumn{5}{|c|}{ 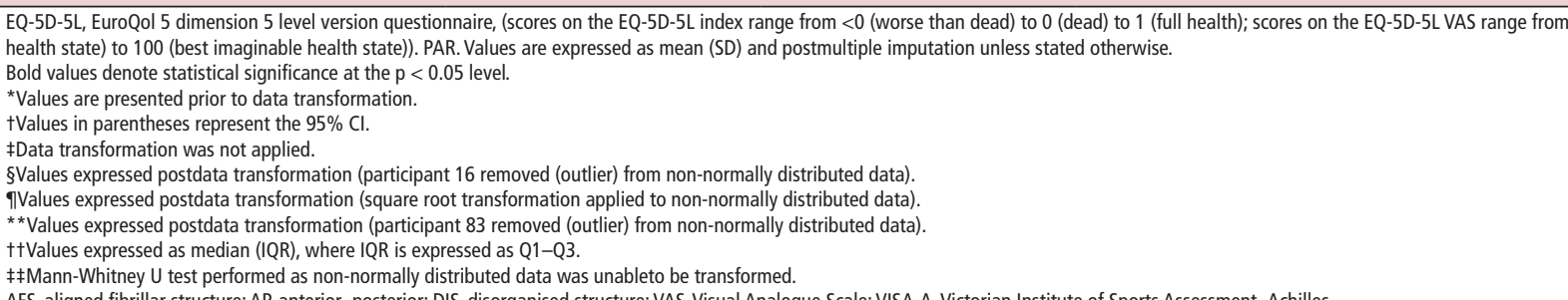 } \\
\hline
\end{tabular}

exercises from a physiotherapist, one reported applying tape and one reported using a topical analgesic/non-steroidal antiinflammatory gel.

\section{Adverse events}

There were no serious adverse events. The most common adverse events were pain in the knee, lower leg and foot and ankle. There were no statistically significant between-group differences in the number of participants reporting adverse events (table 4).

\section{Sensitivity analysis}

Sensitivity analysis was performed to assess the robustness of the findings for the primary outcome measure where multiple imputation was used to handle missing data. We used a series of five alternate approaches to handle missing data (online supplemental file 4). Results demonstrated that the magnitude of difference between groups for the VISA-A ranged from 6.5 to 10.0 points in favour of the heel lifts, with one of the five alternate approaches to handling missing data displaying a statistically significant between-group difference (10.0 points) that could be considered clinically meaningful (online supplemental file 4).

\section{DISCUSSION}

This randomised trial found heel lifts to be more effective in reducing pain and improving function in adults with mid-portion 


\begin{tabular}{|c|c|c|c|c|}
\hline \multirow{2}{*}{$\begin{array}{l}\text { Time period } \\
\text { Weeks }\end{array}$} & \multicolumn{2}{|c|}{ Heel lifts group } & \multicolumn{2}{|c|}{ Eccentric exercise group } \\
\hline & Dayst & $\%$ & Dayst & $\%$ \\
\hline $1-2$ & 7 (6 to 7 ) & 94 & 6 (4 to 6$)$ & 79 \\
\hline $3-6$ & 7 (7 to 7$)$ & 87 & 5 (5 to 7$)$ & 67 \\
\hline $7-12$ & 7 (6 to 7$)$ & 91 & 5 (3 to 5 ) & 60 \\
\hline
\end{tabular}

Days refers to the number of days per week the intervention was reported to be used over the time period.

*Percentage adherence was calculated as follows. For the heel lifts group, the number of days the heel lifts were worn was divided by 7 (ie, the maximum possible number of days per week the heel lifts could be worn) and multiplied by 100 . For the eccentric exercise group, the number of days the eccentric exercise programme was performed was divided by 7 (ie, the maximum possible number of days per week the eccentric exercise programme could be performed) and multiplied by 100 . tValues are expressed as median (IQR), where IQR is expressed as Q1-Q3.

Achilles tendinopathy. This is supported by findings for the primary outcome measure, the VISA-A questionnaire, where a 9.6 point between-group difference was observed in favour of the heel lifts intervention. However, it is unclear if the observed magnitude of difference in the VISA-A is large enough to be clinically meaningful, based on a priori MID of 10 points. The estimated MID for the VISA-A ranges between $6.5^{42}$ and 20 points, ${ }^{43}$ with 10 points the most commonly reported. ${ }^{12} 1316374445$

There were also several important findings for the secondary outcome measures at 12 weeks that favoured the heel lifts intervention, but the clinical importance of the observed differences remains uncertain. First, the severity of pain (measured using a VAS) was significantly less in the heel lifts group, although it is unclear if the magnitude of difference (19 points) is clinically meaningful for mid-portion Achilles tendinopathy. ${ }^{46}$ Second, self-reported treatment effectiveness for both pain and function, measured using the PIC, was significantly greater in participants allocated the heel lifts, with the NNT indicating that four participants would need to be treated with heel lifts to achieve one additional successful outcome. However, the CIs around these values were wide. Third, health-related quality of life (measured using the EQ-5D-5L Index) favoured the heel lifts, but the clinical importance of the observed difference (0.10) is not clear. ${ }^{47}$

\section{Comparison of findings with previous research}

This is the first randomised trial to compare the efficacy of heel lifts to calf muscle eccentric exercise for mid-portion Achilles tendinopathy. Consequently, there are no previous trials with which to compare these findings to. In our study, the mean within-group improvement in VISA-A at 12 weeks was 17.4 points for the eccentric exercise group. The magnitude of improvement is lower than what was reported in a recent systematic review with meta-analysis, where the mean within-group improvement at 12 weeks in VISA-A following an eccentric exercise programme was reported to be 21 points. ${ }^{48}$ However, there was variability in response across included studies, and the authors concluded that the improvement was likely to have been overestimated. Therefore, we believe that our findings regarding the effect of the eccentric exercise programme are valid.

\section{Strengths and limitations}

This randomised trial was pragmatically designed, incorporated rigorous methodology and included two groups with similar baseline characteristics.

Potential confounding factors were controlled for with the use of appropriate participant randomisation and use of ANCOVA statistics for data analysis. Bias was minimised with the use of allocation concealment, double checking of data entry and blinded data analysis. An appropriate sample size to ensure adequate statistical power and the use of diagnostic imaging to confirm mid-portion Achilles tendinopathy of study participants strengthened this trial.

The participants of this study were representative of individuals with Achilles tendinopathy that present to healthcare providers, based on age and sex distribution, although the duration of symptoms of participants of this study (mean 34.4 months) was far greater than previously reported (mean 2.6 and 7.4 months). ${ }^{49} 50$ Further studies are required to test these finding in sub-groups such as younger or highly active individuals. We also used interventions that are readily available and frequently prescribed in clinical practice.

Our results should be interpreted in context of inherent study design limitations. Due to the nature of the interventions, participants and research staff administering the intervention could not be blinded. However, the eccentric exercise programme was the preferred intervention in both groups, and there were no between-group differences in treatment credibility or expectations. Adherence was also self-reported and may not truly reflect actual adherence. Finally, the study duration was 12 weeks, so it remains unknown if the observed effects persist beyond this point.

Table 4 Self-reported adverse events

\begin{tabular}{|c|c|c|c|c|c|c|}
\hline Adverse event* & $\begin{array}{l}\text { Heel lifts group } \\
(n=50) \dagger\end{array}$ & $\begin{array}{l}\text { Eccentric exercise group } \\
(n=50) \dagger\end{array}$ & RR $\ddagger$ & $P$ value & ARI (\%) & NNT-H‡ \\
\hline Developed new pain & $23(46)$ & $22(44)$ & 1.05 (0.68 to 1.61$)$ & 0.841 & $2.0(-16.9$ to 20.7$)$ & 50 (-6 to 5$)$ \\
\hline Upper body & $0(0)$ & $3(6)$ & UTD & UTD & $-6.0(-16.2$ to 2.2$)$ & -17 ( -6 to 47$)$ \\
\hline Lower back & $6(12)$ & $3(6)$ & 2.00 (0.53 to 7.56$)$ & 0.307 & $6.0(-6.1$ to 18.4$)$ & $17(-17$ to 5$)$ \\
\hline Hip & $3(6)$ & $2(4)$ & $1.50(0.26$ to 8.60$)$ & 0.649 & $2.0(-8.3$ to 12.6$)$ & 50 (-12 to 8$)$ \\
\hline Knee & $6(12)$ & $7(14)$ & 0.86 (0.31 to 2.37$)$ & 0.767 & $-2.0(-15.8$ to 11.8$)$ & $-50(-6$ to 9$)$ \\
\hline Lower leg & $8(16)$ & $9(18)$ & 0.89 (0.37 to 2.11$)$ & 0.790 & $-2.0(-16.9$ to 13.0$)$ & -50 (-6 to 8$)$ \\
\hline Foot or ankle§ & $7(14)$ & $9(18)$ & $0.78(0.31$ to 1.93$)$ & 0.587 & $-4.0(-18.6$ to 10.7$)$ & $-25(-5$ to 9$)$ \\
\hline Blister/skin condition & $2(4)$ & $1(2)$ & 2.00 (0.19 to 21.36$)$ & 0.566 & $2.0(-7.0$ to 11.6$)$ & $50(-14$ to 9$)$ \\
\hline
\end{tabular}

Some participants reported more than one adverse event. The heel lifts group was considered the experimental group for all calculations.

*Values are the number of participantswho reported at least one adverse event.

tValues are expressed as $n(\%)$ for each group.

$\neq$ Values in parentheses represent the $95 \% \mathrm{Cl}$.

$\S$ Excludes skin conditions.

ARI, absolute risk increase; NNT-H, number needed to harm; RR, relative risk; ; UTD, unable to determine 


\section{Considerations/implications}

Clinicians should weigh up the potential advantages and disadvantages when deciding to prescribe heel lifts or eccentric exercise for mid-portion Achilles tendinopathy. Both interventions were safe. Eccentric exercise was the preferred intervention, although adherence was lower in participants allocated to this intervention. Poor adherence with eccentric exercise is commonly reported in trials for mid-portion Achilles tendinopathy. ${ }^{1516}$

The difference in adherence between the groups may be explained by the nature of the interventions. The eccentric exercise programme is time-consuming, requires effort and has the potential to elicit discomfort or pain. ${ }^{51}$ In contrast, the heel lifts intervention is convenient as it does not require any effort to obtain therapeutic benefits. Potential disadvantages of heel lifts include costs, ability to use across multiple shoe styles and supply availability.

\section{CONCLUSION}

In this study, heel lifts were more effective than eccentric exercise in reducing pain and improving function at 12 weeks in adults with mid-portion Achilles tendinopathy. Further studies are required to determine if this difference is large enough to be clinically meaningful. Healthcare providers can consider heel lifts as a first-line treatment for mid-portion Achilles tendinopathy.

\section{What are the findings?}

- In-shoe heel lifts are used for mid-portion Achilles tendinopathy, yet evidence from high-quality randomised controlled trials has been lacking.

- We found that heel lifts were effective, yet it remains unknown if heel lifts result in an improvement considered important by patients above the current first-line treatment, eccentric exercise.

\section{How might it impact on clinical practice in the future?}

- Healthcare providers can consider heel lifts as a first-line treatment for mid-portion Achilles tendinopathy.

Twitter Chantel L Rabusin @CRabusin, Hylton B Menz @hyltonmenz, Jodie A McClelland @JodieMcClelland, Angela M Evans @angelamfevans, Peter Malliaras @DrPeteMalliaras, Sean I Docking @SIDocking and Karl B Landorf @karllandorf

Contributors SEM, HBM, KBL, JAM, AME and PM obtained funding for this trial and were responsible for the design of this study. CLR, SID and JMG conducted data collection. CLR, HBM and JMG conducted data analysis and interpreted findings. CLR wrote the initial draft of the manuscript, which was then revised and approved by all authors.

Funding This trial was funded by an Australian Government Research Training Program Scholarship 2015 and a grant from La Trobe University Sport, Exercise and Rehabilitation Research Focus Area (SER RFA Grant Scheme). HBM is currently a National Health and Medical Research Council of Australia Senior Research Fellow (ID: 1135995).

Competing interests None declared.

Patient and public involvement Patients and/or the public were involved in the design, or conduct, or reporting, or dissemination plans of this research. Refer to the Methods section for further details.

Patient consent for publication Not required.

Ethics approval Ethical approval was obtained from the La Trobe University Human Ethics Committee (Application HEC17-064) with written informed consent obtained from all participants. Ethical standards adhered to the National Health and Medical Research Council (NHMRC) National Statement on Ethical Conduct in Human Research.

Provenance and peer review Not commissioned; externally peer reviewed.
Data availability statement Data are available on reasonable request. All data relevant to the study are included in the article or uploaded as online supplementary information. The dataset supporting the conclusions of this article is available on request in the La Trobe University Figshare repository: https://latrobe.figshare.com/.

\section{ORCID iDs}

Chantel L Rabusin http://orcid.org/0000-0001-8111-703X

Hylton B Menz http://orcid.org/0000-0002-2045-3846

Jodie A McClelland http://orcid.org/0000-0002-9317-7571

Angela M Evans http://orcid.org/0000-0002-5527-1161

Peter Malliaras http://orcid.org/0000-0001-5879-4139

Sean I Docking http://orcid.org/0000-0001-7051-7548

Karl B Landorf http://orcid.org/0000-0002-3882-5206

James M Gerrard http://orcid.org/0000-0002-6827-0408

Shannon E Munteanu http://orcid.org/0000-0001-6780-2743

\section{REFERENCES}

1 Järvinen TAH, Kannus P, Maffulli N, et al. Achilles tendon disorders: etiology and epidemiology. Foot Ankle Clin 2005;10:255-66.

2 Bakkegaard M, Johannsen FE, Højgaard B, et al. Ultrasonography as a prognostic and objective parameter in Achilles tendinopathy: a prospective observational study. Eur J Radiol 2015;84:458-62.

3 De Jonge S, Warnaars JLF, De Vos RJ, et al. Relationship between neovascularization and clinical severity in Achilles tendinopathy in 556 paired measurements. Scand J Med Sci Sports 2014;24:773-8.

4 van Dijk CN, van Sterkenburg MN, Wiegerinck Jl, et al. Terminology for Achilles tendon related disorders. Knee Surg Sports Traumatol Arthrosc 2011;19:835-41.

5 Färnqvist K, Malliaras P, Pearson S. Adaptation of tendon structure and function in tendinopathy with exercise and its relationship to clinical outcome. J Sport Rehabil 2019:1-30.

6 Mc Auliffe S, Synott A, Casey H, et al. Beyond the tendon: experiences and perceptions of people with persistent Achilles tendinopathy. Musculoskelet Sci Pract 2017;29:108-14

7 Paavola M, Kannus P, Paakkala T, et al. Long-term prognosis of patients with Achilles tendinopathy. An observational 8-year follow-up study. Am J Sports Med 2000;28:634-42.

8 O'Neill S, Barry S, Watson P. Plantarflexor strength and endurance deficits associated with mid-portion Achilles tendinopathy: the role of soleus. Phys Ther Sport 2019;37:69-76.

9 Ceravolo ML, Gaida JE, Keegan RJ. Quality-Of-Life in Achilles tendinopathy: an exploratory study. Clin J Sport Med 2020;30:495-502.

10 Martin RL, Chimenti R, Cuddeford T, et al. Achilles pain, stiffness, and muscle power deficits: Midportion Achilles tendinopathy revision 2018. J Orthop Sports Phys Ther 2018;48:A1-38.

11 Alfredson $\mathrm{H}$, Pietilä T, Jonsson $\mathrm{P}$, et al. Heavy-load eccentric calf muscle training for the treatment of chronic Achilles tendinosis. Am J Sports Med 1998;26:360-6.

12 Beyer R, Kongsgaard M, Hougs Kjær B, et al. Heavy slow resistance versus eccentric training as treatment for Achilles tendinopathy: a randomized controlled trial. Am J Sports Med 2015;43:1704-11.

13 Silbernagel KG, Thomeé R, Eriksson Bl, et al. Continued sports activity, using a pain-monitoring model, during rehabilitation in patients with Achilles tendinopathy: a randomized controlled study. Am J Sports Med 2007;35:897-906.

14 Couppé C, Svensson RB, Silbernagel KG, et al. Eccentric or concentric exercises for the treatment of tendinopathies? J Orthop Sports Phys Ther 2015;45:853-63.

15 Rompe JD, Nafe B, Furia JP, et al. Eccentric loading, shock-wave treatment, or a wait-and-see policy for tendinopathy of the main body of tendo Achillis: a randomized controlled trial. Am J Sports Med 2007;35:374-83.

16 Sayana MK, Maffulli N. Eccentric calf muscle training in non-athletic patients with Achilles tendinopathy. J Sci Med Sport 2007;10:52-8.

17 Habets B, van Cingel REH. Eccentric exercise training in chronic mid-portion Achilles tendinopathy: a systematic review on different protocols. Scand J Med Sci Sports 2015;25:3-15.

18 Schepsis AA, Jones H, Haas AL. Achilles tendon disorders in athletes. Am J Sports Med 2002;30:287-305.

19 Hunter G. The conservative management of Achilles tendinopathy. Phys Ther Sport 2000;1:6-14.

20 Rabusin CL, Menz HB, McClelland JA, et al. Effects of heel lifts on lower limb biomechanics and muscle function: a systematic review. Gait Posture 2019:69:224-34

21 Rabusin CL, Menz HB, McClelland JA, et al. Efficacy of heel lifts versus calf muscle eccentric exercise for mid-portion Achilles tendinopathy (the HEALTHY trial): study protocol for a randomised trial. J Foot Ankle Res 2019;12:20.

22 Schulz KF, Altman DG, Moher D, et al. CONSORT 2010 statement: updated guidelines for reporting parallel group randomised trials. Int J Surg 2011;9:672-7.

23 Moher D, Hopewell S, Schulz KF, et al. CONSORT 2010 explanation and elaboration: updated guidelines for reporting parallel group randomised trials. Int I Surg 2012;10:28-55. 
24 Hoffmann TC, Glasziou PP, Boutron I, et al. Better reporting of interventions: template for intervention description and replication (TIDieR) checklist and guide. BMJ 2014;348:g1687.

25 The National Health and Medical Research Council, Australian Reserach Council and Universities Australia. National statement on ethical conduct in human research. Canberra: Commonwealth of Australia, 2018.

26 Brewin CR, Bradley C. Patient preferences and randomised clinical trials. BMJ 1989:299:313-5.

27 Devilly GJ, Borkovec TD. Psychometric properties of the credibility/expectancy questionnaire. J Behav Ther Exp Psychiatry 2000;31:73-86.

28 Robinson JM, Cook JL, Purdam C, et al. The VISA-A questionnaire: a valid and reliable index of the clinical severity of Achilles tendinopathy. Br J Sports Med 2001;35:335-41.

29 Docking SI, Cook J. Pathological tendons maintain sufficient aligned fibrillar structure on ultrasound tissue characterization (UTC). Scand J Med Sci Sports 2016:26:675-83.

30 van Schie HTM, de Vos RJ, de Jonge S, et al. Ultrasonographic tissue characterisation of human Achilles tendons: quantification of tendon structure through a novel noninvasive approach. Br J Sports Med 2010;44:1153-9.

31 Dworkin RH, Turk DC, Farrar JT, et al. Core outcome measures for chronic pain clinical trials: IMMPACT recommendations. Pain 2005;113:9-19.

32 Rabin R, de Charro F. EQ-5D: a measure of health status from the EuroQol group. Ann Med 2001;33:337-43.

33 Sallis JF, Haskell WL, Wood PD, et al. Physical activity assessment methodology in the five-city project. Am J Epidemiol 1985;121:91-106.

34 Hébert-Losier K, Wessman C, Alricsson M, et al. Updated reliability and normative values for the standing heel-rise test in healthy adults. Physiotherapy 2017:103:446-52.

35 Collins N, Crossley K, Beller E, et al. Foot orthoses and physiotherapy in the treatment of patellofemoral pain syndrome: randomised clinical trial. Br J Sports Med 2009:43:163-8.

36 Menz HB, Auhl M, Tan JM, et al. Effectiveness of foot orthoses versus rocker-sole footwear for first metatarsophalangeal joint osteoarthritis: randomized trial. Arthritis Care Res 2016;68:581-9.

37 Munteanu SE, Scott LA, Bonanno DR, et al. Effectiveness of customised foot orthoses for Achilles tendinopathy: a randomised controlled trial. Br J Sports Med 2015;49:989-94.

38 Sterne JAC, White IR, Carlin JB, et al. Multiple imputation for missing data in epidemiological and clinical research: potential and pitfalls. BMJ 2009;338:b2393.

39 Bender R, Lange S. Adjusting for multiple testing--when and how? J Clin Epidemiol 2001;54:343-9.
40 Matthews JN, Altman DG, Campbell MJ, et al. Analysis of serial measurements in medical research. BMJ 1990;300:230-5.

41 Twisk J, Proper K. Evaluation of the results of a randomized controlled trial: how to define changes between baseline and follow-up. J Clin Epidemiol 2004;57:223-8.

42 McCormack J, Underwood F, Slaven E, et al. The minimum clinically important difference on the VISA-A and LEFS for patients with insertional Achilles tendinopathy. Int J Sports Phys Ther 2015;10:639-44.

43 Murphy M, Rio E, Debenham J, et al. Evaluating the progress of mid-portion Achilles tendinopathy during rehabilitation: a review of outcome measures for self-reported pain and function. Int J Sports Phys Ther 2018;13:283-92.

44 Bell KJ, Fulcher ML, Rowlands DS, et al. Impact of autologous blood injections in treatment of mid-portion Achilles tendinopathy: double blind randomised controlled trial. BMJ 2013:346:f2310

45 Silbernagel KG, Brorsson A, Lundberg M. The majority of patients with Achilles tendinopathy recover fully when treated with exercise alone: a 5-year follow-up. Am J Sports Med 2011;39:607-13.

46 Hawker GA, Mian S, Kendzerska T, et al. Measures of adult pain: visual analog scale for pain (VAS pain), numeric rating scale for pain (NRS pain), McGill pain questionnaire (MPQ), short-form McGill pain questionnaire (SF-MPQ), chronic pain grade scale (CPGS), short Form-36 bodily pain scale (SF-36 BPS), and measure of intermittent and constant osteoarthritis pain (ICOAP). Arthritis Care Res 2011:63:\$240-52.

47 Coretti S, Ruggeri M, McNamee P. The minimum clinically important difference for EQ-5D index: a critical review. Expert Rev Pharmacoecon Outcomes Res 2014; 14:221-33.

48 Murphy M, Travers M, Gibson W, et al. Rate of improvement of pain and function in mid-portion Achilles tendinopathy with loading protocols: a systematic review and longitudinal meta-analysis. Sports Med 2018;48:1875-91.

49 Albers IS, Zwerver J, Diercks RL, et al. Incidence and prevalence of lower extremity tendinopathy in a Dutch general practice population: a cross sectional study. BMC Musculoskelet Disord 2016;17:16.

50 de Jonge S, van den Berg C, de Vos RJ, et al. Incidence of midportion Achilles tendinopathy in the general population. Br J Sports Med 2011;45:1026-8.

51 Roos EM, Engström M, Lagerquist A, et al. Clinical improvement after 6 weeks of eccentric exercise in patients with mid-portion Achilles tendinopathy -- a randomized trial with 1-year follow-up. Scand J Med Sci Sports 2004;14:286-95. 